\title{
Management of Hepatocellular Carcinoma in Cirrhotic Patients with Portal Hypertension: Relevance of Hagen-Poiseuille's Law
}

\author{
Gerond Lake-Bakaar Muneeb Ahmed Amy Evenson \\ Alan Bonder Salomao Faintuch Vinay Sundaram
}

Liver Tumor Center, Beth Israel Deaconess Medical Center, Harvard Medical School, Boston, Mass., USA

\section{Key Words}

Hagen-Poiseuille's law · Hepatocellular carcinoma · Liver stiffness · Portal hypertension

\begin{abstract}
Background: Hepatic decompensation in cirrhosis heralds an accelerated course with poor survival. An increase in hepatic venous pressure gradient (HVPG), rather than surrogate tests of liver function, appears to be the sole predictor of decompensation after surgical resection. We propose that hepatic sinusoidal walls become less elastic as cirrhosis progresses. Decompensation signals the development of increased vessel wall rigidity. The pressureflow characteristics then become subject to Hagen-Poiseuille's law, which applies only to rigid, cylindrical vessels. Thereafter, HVPG rises exponentially (by a factor inversely proportional to the fourth power of the net radius of functional sinusoidal vessels, $1 / r^{4}$, at any given hepatic blood flow rate. This review attempts to correlate liver stiffness, risk of decompensation and outcomes from hepatocellular carcinoma (HCC) in patients with cirrhosis. Summary: We compare the complexity of autoregulation in the normal elastic liver, which has a unique dual blood supply, with that in the rigid cirrhotic liver. We also review, in the context of background liver cirrhosis, the management of HCC which is in essence, a solid mass of unorganized cells that exacerbates liver stiffness. We discuss the differential effects of various therapeutic modalities such as liver transplantation, loco-regional therapy and drugs on HCC outcomes, based on their effects on HVPG. Key Messages: Increased hepatic artery supply, or the hepatic artery buffer response, may be the only available method for autoregulation or maintenance of hepatic blood flow in the cirrhotic liver. In HCC, loco-regional therapies, including partial resection of the cirrhotic liver, can exacerbate portal hypertension by in-
\end{abstract}


creasing blood flow within the remnant organ. We conclude that studies of HVPG reduction as part of HCC management may be beneficial and are warranted.

Copyright (C) 2014 S. Karger AG, Basel

\section{Introduction}

Functional Hepatic Reserve and Liver Regenerative Potential

The normal healthy liver is well adapted to withstand major damage and injury. This adaptation arises from a large functional redundancy or reserve (FHR) [1, 2] and a significant regenerative potential (RP) [3]. More than $75 \%$ of the normal liver can be surgically resected without risk of liver failure, provided surrogate markers of FHR such as bilirubin and hepatic indocyanine green clearance are normal. As cirrhosis advances, these surrogate markers become less predictive of post-resection decompensation [4].

Cirrhosis occupies a broad, complex, dynamic pathologic spectrum. There are two distinct stages with different prognostic implications: compensated cirrhosis, with a median survival of 12 years, and decompensated cirrhosis (variceal bleeding, hepatic encephalopathy and ascites), with a median survival of only 2 years [5].

In multiple logistic regression analysis, the hepatic venous pressure gradient (HVPG) in the cirrhotic patient stands alone as the only independent variable related to hepatic decompensation following surgical resection [6-12]. This pivotal role of HVPG is unexplained.

Increased HVPG also has an effect on the RP of the cirrhotic liver. In the presence of increased HVPG, resection is associated with the differential expression of genes associated with apoptosis, rather than with regeneration [13]. Several explanations have been proposed. The repeated cell divisions that underlie the process of cirrhosis may lead to senescence and telomere shortening and reduce the RP of the cirrhotic liver [14]. DNA damage checkpoint activation could also reduce the RP of the cirrhotic liver $[15,16]$.

In this review, we propose that, in contrast to the elastic sinusoidal walls in the normal liver, cirrhotic sinusoids are rigid and subject to the Hagen-Poiseuille's law, which applies only to rigid cylindrical vessels. Thus, at constant flow rate, above a threshold of vessel wall rigidity that marks progression from compensated to decompensated cirrhosis,,the sinusoidal pressure gradient rises exponentially (by a factor inversely proportional to the fourth power of the radius, $1 / \mathrm{r}^{4}$ ) with any reduction in the net area or radius of perfused sinusoids that follow surgical resection. The resulting shearing forces induce endothelial damage. Autoregulation, or the hepatic artery buffer response (HABR), is less capable of compensating for this increased pressure gradient and may be a more likely explanation for the reduced RP of the cirrhotic liver $[15,16]$.

\section{Autoregulation of Blood Flow in the Normal and Cirrhotic Liver}

The ability to maintain an adequate blood flow that is consistent with metabolic demands in the face of changes in the inflow perfusion pressure is termed autoregulation. This phenomenon, first described by Johnson et al. for the kidneys, has since been demonstrated in several other organs [17].

Autoregulation: The Normal Liver

Blood flow to organs is classically autoregulated by active changes in the caliber of the inflow arterioles, which in turn decrease or increase capillary blood flow. However, the mi- 
crovasculature of the liver is unique in that there is a dual blood supply. Approximately $80 \%$ originates from the portal venous system at a low pressure of around $15 \mathrm{~mm} \mathrm{Hg}$; the remainder, around 20\%, derives from the hepatic artery at considerably higher pressures (peak 120 $\mathrm{mmHg}$ ) in the terminal hepatic arteriole. The combined systems perfuse the hepatic sinusoids at pressures around 3-6 $\mathrm{mm} \mathrm{Hg}$.

The portal venous system in the normal liver is a passive vascular bed. Active portal venous autoregulation was not observed in the dog [18]. A myogenic response has been described in arterial resistance vessels that control blood flow in the liver [18]. A stepwise reduction in portal venous flow is associated with a simultaneous increase in hepatic arteriolar flow, a phenomenon known as the HABR [19].

The effectiveness of this autoregulation is illustrated by the fact that a $62 \%$ resection of the normal pig liver increases portal venous pressure from $6.1 \mathrm{~mm} \mathrm{Hg}$ to just $8.2 \mathrm{~mm} \mathrm{Hg}$, despite the significantly increased blood flow to the liver remnant. After $75 \%$ resection, portal venous pressure doubles to $12 \mathrm{~mm} \mathrm{Hg}$. It is not until more than $90 \%$ of the liver is resected that a major rise in sinusoidal pressure occurs. The rise in pressure leads to a marked increase in sinusoidal diameter and concomitant histological liver damage [13].

Several compounds have been designated as potentially responsible for liver autoregulation: acetylcholine, endothelium derived relaxing factor (NO), $\mathrm{CO}$, and $\mathrm{H}_{2} \mathrm{~S}$ are possible vasodilators, whereas the three isopeptides of endothelium constricting factor endothelin, ET-1, ET-2 and ET-3, are possible vasoconstrictors. The adenosine washout hypothesis suggests that adenosine might exert physiological control and that the hepatic arterioles dilate when adenosine builds up in the space of Mall. The targets for the candidate compounds include hepatic stellate cells that have a peri-sinusoidal distribution and smooth muscle cells that are located proximal or distal to the hepatic sinusoids.

It is important to note that in the normal liver, hepatic arterial flow may not be crucial or essential for liver viability and may serve largely to buffer the portal venous flow. Thus, acute ligation of the hepatic artery has little impact on liver metabolism. By contrast, when the main portal trunk is ligated, sinusoidal flow is significantly reduced [20].

\section{Autoregulation: The Cirrhotic Liver}

The normal liver has a symmetric architecture allowing blood to flow in an orderly fashion from portal vein and hepatic artery radicals within the portal triads, across the sinusoids to the hepatic vein. By contrast, disorganized nodules disrupt the symmetrical, acinar structure in the cirrhotic liver [21].

Scant data are available on hepatic blood flow in the cirrhotic liver in humans, but some are available in animals. In the $\mathrm{CCl}_{4}$ cirrhotic rat model, total hepatic flow is significantly lower than in normal rats [22]. This results mainly from reduced flow in the low-pressure portal venous system. There is some compensation for this from an increased hepatic artery flow, which doubles its contribution from $20 \%$ in the normal liver to $40 \%$ in the well-compensated cirrhotic liver.

\section{Pressure Gradient in Normal and Cirrhotic Liver}

The Hagen-Poiseuille equation, $\Delta \mathrm{P}=128 \mu \mathrm{LQ} / \pi \mathrm{r}^{4}$, is a physical law in fluid dynamics that governs the pressure gradient $\Delta \mathrm{P}$ in a fluid with viscosity $\mu$ flowing through a rigid cylindrical pipe of length $\mathrm{L}$ and radius $\mathrm{r}$ at volumetric flow rate $\mathrm{Q}$. Thus, the pressure gradient $\Delta \mathrm{P}$ is inversely related to $\mathrm{r}^{4}$, and any change in the net area or radius of perfused sinusoids, will result in exponentially larger changes in the pressure gradient. 


\section{Liver
Cancer}

The normal liver is soft and elastic and the sinusoidal vessels distensible. It is, therefore, not directly subject to the Hagen-Poiseuille law. A passive increase in vessel radius at least partially accommodates any increase in flow and buffers changes in the hepatic venous pressure gradient.

By contrast, the sinusoidal vessel walls become more rigid and attenuated with increasing liver fibrosis and advancing cirrhosis. Thus, at a constant flow rate progressive reduction in net area or radius of sinusoidal vessels with advancing disease would cause the pressure gradient to rise exponentially Similarly, a relatively small increase in sinusoidal blood flow could result in a large increase in the pressure gradient in a cirrhotic liver with rigid vessel walls. Increased HVPG increases sinusoidal shear stress and will worsen liver ischemia.

Large or multifocal hepatocellular carcinomas (HCCs) that comprise nodules of unorganized hepatocytes growing into outflow vessels and surrounded by inflammatory cells can also increase liver stiffness. Liver stiffness can be objectively measured using a FibroScan [23] and is an independent predictor of HCC outcome [24]. Liver elasticity can be independently assessed using magnetic resonance elastography or elastography. A number of recent studies have shown that transient elastography correlates well with HVPG [12, 25]. These tests could prove more predictive of post-resection outcomes than current functional tests [10]. Meta-analysis of studies of liver stiffness suggests an association with risk of decompensation, liver cancer and death in patients with chronic liver disease [26].

Management of HCC in the Cirrhotic Liver: Significance of Liver Stiffness or Portal Hypertension

HCC is a leading cause of cancer-related deaths worldwide, accounting for more than 600,000 deaths each year [27]. In the United States, the incidence has tripled over the past three decades. It is estimated that more than 20,000 cases are diagnosed each year [27].

In the second part of this review, we discuss the current management of HCC in the context of portal hypertension. Loco-regional therapy, including surgical resection, reduces the net surface area or radius of functioning sinusoids in the remnant liver. The impact of net sinusoidal radius $r^{4}$ of the liver remnant correlates with the volume of liver removed. This has greatest significance for the stiff liver and correlates with outcome.

Liver Transplantation versus Surgical Resection for HCC

In patients with compensated or decompensated cirrhosis and early HCC that falls within the Milan criteria [28], liver transplantation probably offers the best survival outcome $[29,30]$. Based on intention-to-treat analysis, 1-, 3- and 5- year survival after liver transplantation has been reported as $85 \%, 69 \%$ and $69 \%$ [31]. The availability of donor livers is the most important limitation [31]. Liver transplantation is a better alternative than resection in patients with underlying portal hypertension. The liver graft in the immediate posttransplantation period has characteristics suggestive of a stiff liver. Viability is dependent on blood supply from the hepatic artery [32].

\section{Surgical Resection}

Surgical resection is a therapeutic alternative to transplantation in HCC management [33]. However, resection is frequently associated with decompensation in patients with evidence of portal hypertension and increased HVPG [34]. This severely restricts the number of patients that can be treated with resection [35].

The peri-operative mortality for surgical resection in experienced centers is less than $3 \%$ and the 5 -year survival rate is $70 \%$ or more. However, there is increased risk of tumor recurrence in the diseased liver that can exceed $50 \%$ at 5 years $[36,37]$. DNA microarray and other studies suggest that up to $70 \%$ of these tumor recurrences are intrahepatic metastases 
and only $30 \%$ truly arise de novo [38]. There is also the risk of incomplete resection, because surgery tends to be conservative as a result of the reduced FHR and RP in the cirrhotic liver.

After liver resection, the net available cross-sectional area or radius of perfusable sinusoids $\mathrm{r}^{4}$ in the remnant liver is reduced. In the stiff cirrhotic liver, this can translate into an acute rise in the pressure gradient. The damage induced by the ensuing shearing forces within the sinusoids is more marked in patients with pre-existing portal hypertension. Therefore, unlike transplantation, resection is limited to cirrhotic patients without existing evidence of increased HVPG or hepatic decompensation.

In preparation for resection, portal vein embolization has been used successfully for many years to induce hypertrophy and hyperplasia of the future liver remnant $[39,40]$. Although, less effective for the advanced cirrhotic liver [41], the reduction in net radius of perfused sinusoids $\mathrm{r}^{4}$ is attenuated, as is the subsequent rise in pressure gradient. We propose that mechanisms for reducing HVPG in the remnant liver such as artificial shunting or drugs that can reduce sinusoidal pressure might also reduce the risk of decompensation.

\section{Locoregional Therapy: Influence of Portal Hypertension}

Locoregional therapy involves techniques for local control of tumor growth. Currently, common modalities for treatment include tumor ablation (either percutaneous or laparoscopic) [42], transcatheter intra-arterial therapies (including injection of chemotherapy or radiation-emitting beads, often with additional embolization/occlusion of arterial flow) $[43,44]$ and targeted ablative radiation (i.e., stereotactic radiotherapy or CyberKnife). The goal of these therapies is to induce localized, targeted destruction or injury to tumor while minimizing injury to surrounding liver. Additionally, loco-regional procedures are largely done with some form of imaging guidance and in a minimally invasive manner.

The influence of liver stiffness, portal hypertension and HVPG on the outcomes of locoregional therapies depends in large part on the volume of liver damaged or removed. Detailed descriptions of the techniques and clinical outcomes for locoregional therapies fall outside the scope of this article, but are readily available in the literature.

\section{Tumor Ablation}

Tumor ablation with image-guidance via percutaneous or laparoscopic approaches induces local tissue injury and cell death. Ablation treats focal HCC in a very targeted manner. The planned area of ablation includes a 5- to $10-\mathrm{mm}$ rim of surrounding normal tissue to achieve an ablative margin. Detailed reviews of the principles of ablation are readily available in the literature $[45,46]$.

Several ablative modalities are in current clinical use. High-temperature radiofrequency (RF) ablation platforms, where temperatures of $>60^{\circ} \mathrm{C}$ are induced throughout the target area, are by far the most commonly used in HCC. Long-term survival in patients with small tumors $(<3 \mathrm{~cm})$, well-compensated (Childs A) cirrhosis and normal or marginally elevated portal pressures approaches $70 \%$ in experienced centers [46-49]. Chemical ablation with ethanol injection, while previously used commonly for HCC, is now reserved for lesions not amenable to thermal ablation. Other newer [50] modalities, such as ultrasound-based thermal ablation (i.e., High-intensity focused ultrasound) or non-thermal irreversible electroporation are still largely experimental.

The current clinical guidelines restrict tumor ablation to focal tumors $3-5 \mathrm{~cm}$ in diameter. Therefore, the impact of these small changes in sinusoidal vessel area or radius in the liver remnant and consequently on HVPG is likely to be small, even when multiple tumors are ablated simultaneously. This is borne out by the results from very large studies that have dem- 
onstrated that tumor ablation is well tolerated, with little to no effect on FHR or surrogates of portal hypertension such as ascites or post-procedural variceal bleeding [50-53].

Small segmental and branch portal venous thrombosis is sometimes seen after adjacent RF ablation and could conceivably increase local portal pressure. However, the incidence of this is fairly low, because blood flow protects vascular structures by removing heat from the ablation zone (the heat-sink effect). Reports of occlusion of large branches or of the main portal vein from thermal ablation are rare [54]. Theoretically at least, increased portal hypertension can, by enlarging segmental portal veins, remove heat from the ablation zone [55] and contribute to incomplete tumor ablation [56]. Newer ablative modalities, such as microwave-based platforms that can achieve higher tissue temperatures, are less susceptible to the heat-sink effect [57].

\section{Transcatheter Intra-Arterial Therapies}

Transcatheter intra-arterial therapies for HCC predominantly include chemoembolization (TACE) using a mixture of chemotherapy emulsified in lipiodol (also known as conventional TACE or lipiodol-TACE) or loaded in drug-eluting beads (DEB-TACE) [43]. The rationale for TACE is based on the difference in blood supply between HCC tumors and normal livers. HCC tumors recruit their blood supply and nutrients exclusively from the hepatic arterial supply, whereas both hepatic arterial and portal venous blood can supply native liver. Additionally, HCC tumors are largely hypervascular relative to surrounding liver, which leads to preferential drug and embolic agent flow-based delivery to tumor rather than to liver. TACE techniques vary considerably as described in the literature. To date, no differences in outcomes have been identified between multi-drug chemotherapy and single-drug chemotherapy [58] or among embolization techniques. Transarterial embolization (TAE, or "bland embolization") without chemotherapy is not as effective as TACE [59] and has not demonstrated survival benefit [60]. Radioembolization with yittrium-90-labelled beads can also be performed for HCC, although it is less commonly used than TACE.

Two randomized controlled studies have demonstrated improved survival in patients treated with TACE compared to best supportive care $[61,62]$. TACE is considered the standard of care for patients with unresectable intermediate-stage HCC [63].

Few studies have investigated the effects of TACE on portal hemodynamics and HPVG. Spahr et al. studied acute changes in portal flow velocity (PFV) using Doppler ultrasonography as a surrogate for HPVG at 2 and $24 \mathrm{~h}$ after selective TACE for HCC [64]. In 15 patients, increased portal flow velocities were observed in both the embolized and non-embolized lobes at $2 \mathrm{~h}$ after TACE, with a persistently elevated portal flow velocity PFV in the embolized lobe only at $24 \mathrm{~h}$. The authors suggest that, because the increase in PFV after super-selective TACE involves both the embolized and non-embolized lobes, mechanisms must exist in the cirrhotic patient for the regulation of portal and hepatic arterial hemodynamics [64]. However, as discussed earlier, there is currently no evidence for autoregulation of the portal venous inflow in either normal [18] or cirrhotic animals [22]. A more likely explanation is a passive increase in total portal venous flow, because reducing the flow from the high pressure arterial circuit automatically reduces ambient HVPG.

Increases in baseline liver function tests and total bilirubin are commonly observed after TACE and are consistent with collateral damage. Indirect surrogates of worsening portal hemodynamics, such as increased ascites, have also been observed, reflecting increased portal pressure. Variceal bleeding after TACE is rare and occurs in less than 1\% of cases. Ascites, alpha-fetoprotein and alkaline phosphatase levels are the only true correlates with prognosis [65].

The subset of HCC patients undergoing TACE with transjugular intrahepatic porto-systemic shunts (TIPS) in place represents an interesting model for studying the role of HVPG. 
These patients already have decompensated liver disease and would be expected to decompensate further. Although only a few studies are available, most suggest that TIPS does not adversely affect TACE outcome [66-70]. A positive spin might be that TIPS prevented the expected deterioration.

A recent retrospective analysis of 158 patients undergoing TACE, 10 with previous TIPS, suggested a higher incidence of serious hepato-biliary adverse events in TIPS patients [71], which is unexplained. However, there was no difference in the 1-year survival between the two groups, indicating the presence of long-term compensatory mechanisms.

Further studies relating outcomes after loco-regional therapies to liver stiffness or elasticity directly and longitudinally are indicated. From our discussion above, the concomitant use of drugs (such as sorafenib) or procedures (such as TIPs) that reduce portal pressure might reduce the risk of post-TACE decompensation.

\section{CyberKnife}

CyberKnife, or stereotactic body radiation therapy, limits exposure of non-tumor cells to radiation damage by focusing ionizing radiation on target tissue even during motion of the liver with respiration. It has been shown to be safe and effective for primary HCC [72] and is useful as a bridge prior to transplantation [73]. An advantage over TACE is that it produces acceptable local control in patients with portal vein tumor thrombosis [74]. A comparison between CyberKnife and RFA or TACE on liver stiffness is indicated.

\section{Systemic Therapy - Sorafenib}

Sorafenib is an oral multi-kinase inhibitor that inhibits cell proliferation and angiogenesis. It targets several tyrosine kinases such as Raf kinase and Vascular Endothelial Growth Factor (VEGF) receptor 2 and 3 as well as Platelet-derived Growth Factor (PDGF) receptor beta. It is the only drug currently approved for the treatment of HCC [75].

An interesting and potentially important observation from animal models is that sorafenib reduces developing and established portal hypertension [76-78]. Two recent studies in patients with cirrhosis and HCC have demonstrated a decrease in portal venous flow or pressure on sorafenib treatment $[79,80]$, with a trend toward better survival in those patients with reduced HVPG (20.5 vs 10.6 months). Two of the four responders received concomitant beta-blockers.

The effect of sorafenib on portal venous flow and portal pressure in patients with cirrhosis and HCC deserves further study. In trials in renal cell carcinoma (RCC), an increased risk of gastrointestinal bleeding was observed [75]. By contrast, in HCC and cirrhosis trials, bleeding risk was similar to that for a placebo. This is surprising, because patients with cirrhosis frequently have thrombocytopenia, increased prothrombin times, varices and congestive gastropathy and would be assumed to be at greater risk of bleeding than patients with RCC. Sorafenib might exert a protective effect against bleeding through a reduction of portal pressure.

In the pivotal trial of sorafenib in HCC [75], patients with liver tumor arising within a background of Hepatitis $\mathrm{C}$ virus (HCV) cirrhosis fared better than those with chronicHepatitis B virus (HBV) or other chronic liver disorders. A possible explanation is that, unlike HBVrelated HCC that can arise in non-cirrhotic livers, HCV-infected patients are almost invariably cirrhotic [81]. Improved survival in these patients might reflect an effect of sorafenib on portal pressure.

Finally, a trend toward improved survival was observed in HCC patients treated with sorafenib who had reductions in HPVG [80]. A combination of sorafenib plus propranolol, nadolol or carvedilol, obeticholic acid or statins in HCC patients might prove useful in increasing patient survival or in reducing the risk of decompensation after resection or TACE. 


\section{Summary}

The backdrop of cirrhosis complicates the management of HCC. Scarring in the cirrhotic liver reduces elasticity and increases liver stiffness. Nodules of HCC cells disorganize the blood flow through the liver, further aggravating liver stiffness. With increased stiffness, the sinusoidal vessel walls become more rigid and, in accordance with Hagen-Poiseuille's law, HVPG increases very steeply with any change in net area or radius of perfused sinusoidal vessels, which follow resection or other locoregional therapy. The shearing forces consequent upon increasing HVPG can lead to severe damage to endothelial cells. HCC progression and management is therefore dependent on liver stiffness and its surrogate, HVPG. Studies designed to investigate correlations between liver elasticity, treatment modality and outcomes are warranted. Trials combining the various forms of loco-regional therapy with portal pressure reduction using drugs, such as sorafenib, propranolol, nadolol or carvedilol, obeticholic acid and statins, or procedures such as TIPS are also warranted.

\section{Acknowledgment}

Authorship Statement: Dr. Gerond Lake-Bakaar is the submission's guarantor (i.e., the person who takes responsibility for the integrity of the work as a whole, from inception to published article.) Dr. Lake-Bakaar was responsible for the original conception and preparation of the manuscript. The coauthors contributed equally to the work, applying their areas of specialty to the core hypothesis or researching articles for the work. The authors do not have any financial disclosures or conflicts of interest. All authors approved the final version of the manuscript.

\section{References}

1 Hur H, Ko YT, Min BS, Kim KS, Choi JS, Sohn SK, Cho CH, Ko HK, Lee JT, Kim NK: Comparative study of resection and radiofrequency ablation in the treatment of solitary colorectal liver metastases. Am J Surg 2009;197:728-736.

2 MacIntosh EL, Minuk GY: Hepatic resection in patients with cirrhosis and hepatocellular carcinoma. Surg Gynecol Obstet 1992;174:245-254.

-3 Hammond JS, Guha IN, Beckingham IJ, Lobo DN: Prediction, prevention and management of postresection liver failure. Br J Surg 2011;98:1188-1200.

4 Manizate F, Hiotis SP, Labow D, Roayaie S, Schwartz M: Liver functional reserve estimation: state of the art and relevance to local treatments. Oncology 2010;78(Suppl 1):131-134.

5 D’Amico G, Garcia-Tsao G, Pagliaro L: Natural history and prognostic indicators of survival in cirrhosis: a systematic review of 118 studies. J Hepatol 2006;44:217-231.

6 Bosch J, Abraldes JG, Berzigotti A, Garcia-Pagan JC: The clinical use of HVPG measurements in chronic liver disease. Nature reviews. Gastroenterol Hepatol 2009;6:573-582.

7 Stremitzer S, Tamandl D, Kaczirek K, Maresch J, Abbasov B, Payer BA, Ferlitsch A, Gruenberger T: Value of hepatic venous pressure gradient measurement before liver resection for hepatocellular carcinoma. Br J Surg 2011;98:1752-1758.

-8 Figueras J, Llado L, Ruiz D, Ramos E, Busquets J, Rafecas A, Torras J, Fabregat J: Complete versus selective portal triad clamping for minor liver resections: a prospective randomized trial. Ann Surg 2005;241:582590.

9 An M, Park JW, Shin JA, Choi JI, Kim TH, Kim SH, Lee WJ, Park SJ, Hong EK, Kim CM: [The adverse effect of indirectly diagnosed portal hypertension on the complications and prognosis after hepatic resection of hepatocellular carcinoma]. Korean J Hepatol 2006;12:553-561.

10 Llop E, Berzigotti A, Reig M, Erice E, Reverter E, Seijo S, Abraldes JG, Bruix J, Bosch J, García-Pagan JC: Assessment of portal hypertension by transient elastography in patients with compensated cirrhosis and potentially resectable liver tumors. J Hepatol 2012;56:103-108.

-11 Blasco A, Forns X, Carrión JA, García-Pagán JC, Gilabert R, Rimola A, Miquel R, Bruguera M, García-Valdecasas JC, Bosch J, Navasa M: Hepatic venous pressure gradient identifies patients at risk of severe hepatitis C recurrence after liver transplantation. Hepatology 2006;43:492-499.

12 Castera L, Pinzani M, Bosch J: Non invasive evaluation of portal hypertension using transient elastography. J Hepatol 2012;56:696-703. 
13 Mortensen KE, Conley LN, Hedegaard J, Kalstad T, Sorensen P, Bendixen C, Revhaug A: Regenerative response in the pig liver remnant varies with the degree of resection and rise in portal pressure. Am J Physiol Gastrointest Liver Physiol 2008;294:G819-G830.

14 El-Serag HB, Rudolph KL: Hepatocellular carcinoma: epidemiology and molecular carcinogenesis. Gastroenterology 2007;132:2557-2576.

15 Merle P, Trepo C: Molecular mechanisms underlying hepatocellular carcinoma. Viruses 2009;1:852-872.

16 Daugherity EK, Balmus G, Al Saei A, Moore ES, Abi Abdallah D, Rogers AB, Weiss RS, Maurer KJ: The DNA damage checkpoint protein ATM promotes hepatocellular apoptosis and fibrosis in a mouse model of nonalcoholic fatty liver disease. Cell Cycle 2012;11:1918-1928.

17 Stainsby WN: Local control of regional blood flow. Annu Rev Physiol 1973;35:151-168.

18 Hanson KM, Johnson PC: Local control of hepatic arterial and portal venous flow in the dog. Am J Physiol 1966;211:712-720.

19 McCuskey RS: Morphological mechanisms for regulating blood flow through hepatic sinusoids. Liver 2000;20:3-7.

20 Oda M, Yokomori H, Han JY: Regulatory mechanisms of hepatic microcirculatory hemodynamics: hepatic arterial system. Clin Hemorheol Microcirc 2006;34:11-26.

21 Onori P, Morini S, Franchitto A, Sferra R, Alvaro D, Gaudio E: Hepatic microvascular features in experimental cirrhosis: a structural and morphometrical study in CCl4-treated rats. J Hepatol 2000;33:555-563.

22 Richter S, Mücke I, Menger MD, Vollmar B: Impact of intrinsic blood flow regulation in cirrhosis: maintenance of hepatic arterial buffer response. Am J Physiol Gastrointest Liver Physiol 2000;279:G454-G462.

23 Cescon M, Colecchia A, Cucchetti A, Peri E, Montrone L, Ercolani G, Festi D, Pinna AD: Value of transient elastography measured with FibroScan in predicting the outcome of hepatic resection for hepatocellular carcinoma. Ann Surg 2012;256:706-712, discussion 712-713.

-24 Kuo YH, Lu SN, Hung CH, Kee KM, Chen CH, Hu TH, Lee CM, Changchien CS, Wang JH: Liver stiffness measurement in the risk assessment of hepatocellular carcinoma for patients with chronic hepatitis. Hepatology International 2010;4:700-706.

25 Shi KQ, Fan YC, Pan ZZ, Lin XF, Liu WY, Chen YP, Zheng MH. Transient elastography: a meta-analysis of diagnostic accuracy in evaluation of portal hypertension in chronic liver disease. Liver International 2013;33:62-71.

-26 Singh S, Fujii LL, Murad MH, Wang Z, Asrani SK, Ehman RL, Kamath PS, Talwalkar JA. Liver stiffness is associated with risk of decompensation, liver cancer, and death in patients with chronic liver diseases: a systematic review and meta-analysis. Clinical Gastroenterology and Hepatology 2013;11:1573-1584 e2.

27 El-Serag HB, Mason AC: Rising incidence of hepatocellular carcinoma in the United States. N Engl J Med 1999;340:745-750.

28 Hanish SI, Knechtle SJ: Liver transplantation for the treatment of hepatocellular carcinoma. Oncol Williston Park 2011;25:752-757.

29 Lee Cheah Y, K H Chow P: Liver transplantation for hepatocellular carcinoma: an appraisal of current controversies. Liver Cancer 2012;1:183-189.

30 Chan SC: Liver transplantation for hepatocellular carcinoma. Liver Cancer 2013;2:338-344.

31 Llovet JM, Fuster J, Bruix J: Intention-to-treat analysis of surgical treatment for early hepatocellular carcinoma: resection versus transplantation. Hepatology 1999;30:1434-1440.

-32 Tzakis AG, Gordon RD, Shaw BW Jr, Iwatsuki S, Starzl TE: Clinical presentation of hepatic artery thrombosis after liver transplantation in the cyclosporine era. Transplantation 1985;40:667-671.

-33 Belghiti J, Fuks D: Liver resection and transplantation in hepatocellular carcinoma. Liver Cancer 2012;1:7182.

-34 Chan KM, Lee CF, Wu TJ, Chou HS, Yu MC, Lee WC, Chen MF: Adverse outcomes in patients with postoperative ascites after liver resection for hepatocellular carcinoma. World J Surg 2012;36:392-400.

-35 Helling TS, Woodall CE, 3rd: Referrals for surgical therapy in patients with hepatocellular carcinoma: a community experience. Journal of Gastrointestinal Surgery 2007;11:76-81.

-36 Bolondi L, Sofia S, Siringo S, Gaiani S, Casali A, Zironi G, Piscaglia F, Gramantieri L, Zanetti M, Sherman M: Surveillance programme of cirrhotic patients for early diagnosis and treatment of hepatocellular carcinoma: a cost effectiveness analysis. Gut 2001;48:251-259.

37 Bruix J, Castells A, Bosch J, Feu F, Fuster J, Garcia-Pagan JC, Visa J, Bru C, Rodés J: Surgical resection of hepatocellular carcinoma in cirrhotic patients: prognostic value of preoperative portal pressure. Gastroenterology 1996;111:1018-1022.

38 Ng IO, Guan XY, Poon RT, Fan ST, Lee JM: Determination of the molecular relationship between multiple tumour nodules in hepatocellular carcinoma differentiates multicentric origin from intrahepatic metastasis. J Pathol 2003;199:345-353.

-39 Chik BH, Liu CL, Fan ST, Lo CM, Poon RT, Lam CM, Wong J: Tumor size and operative risks of extended rightsided hepatic resection for hepatocellular carcinoma: implication for preoperative portal vein embolization. Arch Surg 2007;142:63-69, discussion 69.

40 Wakabayashi H, Yachida S, Maeba T, Maeta H: Indications for portal vein embolization combined with major hepatic resection for advanced-stage hepatocellular carcinomas. A preliminary clinical study. Dig Surg 2000;17:587-594.

41 Abdalla EK, Hicks ME, Vauthey JN: Portal vein embolization: rationale, technique and future prospects. $\mathrm{Br}$ J Surg 2001;88:165-175.

42 Lin SM: Local ablation for hepatocellular carcinoma in Taiwan. Liver Cancer 2013;2:73-83.

-43 Lencioni R: Chemoembolization in patients with hepatocellular carcinoma. Liver Cancer 2012;1:41-50. 
44 Kudo M: Treatment of advanced hepatocellular carcinoma with emphasis on hepatic arterial infusion chemotherapy and molecular targeted therapy. Liver Cancer 2012;1:62-70.

-45 Ahmed M, Brace CL, Lee FT Jr, Goldberg SN: Principles of and advances in percutaneous ablation. Radiology 2011;258:351-369.

46 Lencioni R, Crocetti L: Local-regional treatment of hepatocellular carcinoma. Radiology 2012;262:43-58.

-47 Lencioni R, Cioni D, Crocetti L, Bartolozzi C: Percutaneous ablation of hepatocellular carcinoma: state-ofthe-art. Liver Transplantation 2004;10:S91-97.

48 Lencioni R, Crocetti L: Image-guided thermal ablation of hepatocellular carcinoma. Crit Rev Oncol Hematol 2008;66:200-207.

49 Omata M, Tateishi R, Yoshida H, Shiina S: Treatment of hepatocellular carcinoma by percutaneous tumor ablation methods: Ethanol injection therapy and radiofrequency ablation. Gastroenterology 2004;127(Suppl 1):S159-S166.

50 Livraghi T, Meloni F, Solbiati L, Zanus G, Collaborative Italian Group using AMICA system: Complications of microwave ablation for liver tumors: results of a multicenter study. Cardiovasc Intervent Radiol 2012;35:868-874.

51 Bertot LC, Sato M, Tateishi R, Yoshida H, Koike K: Mortality and complication rates of percutaneous ablative techniques for the treatment of liver tumors: a systematic review. Eur Radiol 2011;21:2584-2596.

52 Livraghi T, Solbiati L, Meloni MF, Gazelle GS, Halpern EF, Goldberg SN: Treatment of focal liver tumors with percutaneous radio-frequency ablation: complications encountered in a multicenter study. Radiology 2003;226:441-451.

53 Rhim H, Yoon KH, Lee JM, Cho Y, Cho JS, Kim SH, Lee WJ, Lim HK, Nam GJ, Han SS, Kim YH, Park CM, Kim PN, Byun JY: Major complications after radio-frequency thermal ablation of hepatic tumors: spectrum of imaging findings. Radiographics 2003;23:123-134; discussion 134-136.

54 Francica G, Marone G, Solbiati L, D’Angelo V, Siani A: Hemobilia, intrahepatic hematoma and acute thrombosis with cavernomatous transformation of the portal vein after percutaneous thermoablation of a liver metastasis. Eur Radiol 2000;10:926-929.

55 Lu DS, Raman SS, Limanond P, Aziz D, Economou J, Busuttil R, Sayre J: Influence of large peritumoral vessels on outcome of radiofrequency ablation of liver tumors. J Vasc Interv Radiol 2003;14:1267-1274.

56 Sheiman RG, Mullan C, Ahmed M: In vivo determination of a modified heat capacity of small hepatocellular carcinomas prior to radiofrequency ablation: correlation with adjacent vasculature and tumour recurrence. International Journal of Hyperthermia 2012;28:122-131.

57 Zhou WP, Lai EC, Li AJ, Fu SY, Zhou JP, Pan ZY, Lau WY, Wu MC: A prospective, randomized, controlled trial of preoperative transarterial chemoembolization for resectable large hepatocellular carcinoma. Ann Surg 2009;249:195-202.

58 Sahara S, Kawai N, Sato M, Tanaka T, Ikoma A, Nakata K, Sanda H, Minamiguchi H, Nakai M, Shirai S, Sonomura T: Prospective evaluation of transcatheter arterial chemoembolization (TACE) with multiple anti-cancer drugs (epirubicin, cisplatin, mitomycin c, 5-fluorouracil) compared with TACE with epirubicin for treatment of hepatocellular carcinoma. Cardiovasc Intervent Radiol 2012;35:1363-1371.

-59 Malagari K, Pomoni M, Spyridopoulos TN, Moschouris H, Kelekis A, Dourakis S, Alexopoulou E, Koskinas J, Angelopoulos M, Kornezos J, Pomoni A, Tandeles S, Marinis A, Rizos S, Kelekis D: Safety profile of sequential transcatheter chemoembolization with DC Bead ${ }^{\mathrm{TM}}$ : results of 237 hepatocellular carcinoma (HCC) patients. Cardiovasc Intervent Radiol 2011;34:774-785.

60 Bruix J, Llovet JM, Castells A, Montañá X, Brú C, Ayuso MC, Vilana R, Rodés J: Transarterial embolization versus symptomatic treatment in patients with advanced hepatocellular carcinoma: results of a randomized, controlled trial in a single institution. Hepatology 1998;27:1578-1583.

-61 Sun PL, Chen CL, Hsu SL, Huang TL, Chen TY, Chen YS, Tsang LC, Cheng YF: The significance of transarterial embolization for advanced hepatocellular carcinoma in liver transplantation. Transplant Proc 2004;36:2295-2296.

62 Llovet JM: Evidence-based medicine in the treatment of hepatocellular carcinoma. J Gastroenterol Hepatol 2002;17(Suppl 3):S428-S433.

63 Forner A, Llovet JM, Bruix J: Chemoembolization for intermediate HCC: is there proof of survival benefit? J Hepatol 2012;56:984-986.

64 Spahr L, Becker C, Pugin J, Majno PE, Hadengue A: Acute portal hemodynamics and cytokine changes following selective transarterial chemoembolization in patients with cirrhosis and hepatocellular carcinoma. Med Sci Monit 2003;9:CR383-CR388.

65 Dvorchik I, Carr BI: A simple prognostic scoring system for patients with unresectable hepatocellular carcinoma treated by chemo-embolization. Cancer Detect Prev 2007;31:154-160.

66 Kim TW, Kim HJ, Chon CU, Won HS, Park JH, Park DI, Cho YK, Sohn CI, Jeon WK, Kim BI: Is there any vindication for low dose nonselective beta-blocker medication in patients with liver cirrhosis? Clinical and Molecular Hepatology 2012;18:203-212.

67 Tesdal IK, Wikström M, Flechtenmacher C, Filser T, Dueber C: Percutaneous treatment of hepatocellular carcinoma in patients with transjugular intrahepatic portosystemic shunts. Cardiovasc Intervent Radiol 2006;29:778-784.

68 Sakaguchi H, Uchida H, Maeda M, Matsuo N, Kichikawa K, Ohishi H, Nishida H, Ueno K, Nishimine K, Rösch $\mathrm{J}$, et al: Combined transjugular intrahepatic portosystemic shunt and segmental Lipiodol hepatic artery embolization for the treatment of esophagogastric varices and hepatocellular carcinoma in patients with cirrhosis: preliminary report. Cardiovasc Intervent Radiol 1995;18:9-15. 
69 Tazawa J, Sakai Y, Yamane M, Kakinuma S, Maeda M, Suzuki K, Miyasaka Y, Nagayama K, Kusano F, Sato C: Long-term observation after transjugular intrahepatic portosystemic stent-shunt in two patients with hepatocellular carcinoma. J Clin Gastroenterol 2000;31:262-267.

70 Nicolini A, Saccheri S, Lovaria A, Maggi A, Cazzaniga M, Panzeri A, Salerno F: Prevention of variceal rebleeding and treatment of liver carcinoma by consecutive transjugular intrahepatic portosystemic shunt and hepatic artery chemoembolization. Ital J Gastroenterol 1996;28:269-271.

-71 Kohi MP, Fidelman N, Naeger DM, LaBerge JM, Gordon RL, Kerlan RK Jr: Hepatotoxicity after transarterial chemoembolization and transjugular intrahepatic portosystemic shunt: do two rights make a wrong? J Vasc Interv Radiol 2013;24:68-73.

72 Huang WY, Jen YM, Lee MS, Chang LP, Chen CM, Ko KH, Lin KT, Lin JC, Chao HL, Lin CS, Su YF, Fan CY, Chang YW: Stereotactic body radiation therapy in recurrent hepatocellular carcinoma. Int J Radiat Oncol Biol Phys 2012;84:355-361.

73 O'Connor JK, Trotter J, Davis GL, Dempster J, Klintmalm GB, Goldstein RM: Long-term outcomes of stereotactic body radiation therapy in the treatment of hepatocellular cancer as a bridge to transplantation. Liver Transplantation 2012;18:949-954.

74 Choi BO, Choi IB, Jang HS, Kang YN, Jang JS, Bae SH, Yoon SK, Chai GY, Kang KM: Stereotactic body radiation therapy with or without transarterial chemoembolization for patients with primary hepatocellular carcinoma: preliminary analysis. BMC Cancer 2008;8:351.

75 Llovet JM, Ricci S, Mazzaferro V, Hilgard P, Gane E, Blanc JF, de Oliveira AC, Santoro A, Raoul JL, Forner A, Schwartz M, Porta C, Zeuzem S, Bolondi L, Greten TF, Galle PR, Seitz JF, Borbath I, Häussinger D, Giannaris T, Shan M, Moscovici M, Voliotis D, Bruix J, SHARP Investigators Study Group: Sorafenib in advanced hepatocellular carcinoma. N Engl J Med 2008;359:378-390.

-76 Hennenberg M, Trebicka J, Stark C, Kohistani AZ, Heller J, Sauerbruch T: Sorafenib targets dysregulated Rho kinase expression and portal hypertension in rats with secondary biliary cirrhosis. Br J Pharmacol 2009;157:258-270.

77 Mejias M, Garcia-Pras E, Tiani C, Miquel R, Bosch J, Fernandez M: Beneficial effects of sorafenib on splanchnic, intrahepatic, and portocollateral circulations in portal hypertensive and cirrhotic rats. Hepatology 2009;49:1245-1256.

78 Reiberger T, Rasoul-Rockenschaub S, Rieger A, Ferenci P, Gangl A, Peck-Radosavljevic M: Efficacy of interferon in immunocompromised HCV patients after liver transplantation or with HIV co-infection. Eur J Clin Invest 2008;38:421-429.

79 Coriat R, Gouya H, Mir O, Ropert S, Vignaux O, Chaussade S, Sogni P, Pol S, Blanchet B, Legmann P, Goldwasser F: Reversible decrease of portal venous flow in cirrhotic patients: a positive side effect of sorafenib. PLoS ONE 2011;6:e16978.

80 Pinter M, Sieghart W, Reiberger T, Rohr-Udilova N, Ferlitsch A, Peck-Radosavljevic M: The effects of sorafenib on the portal hypertensive syndrome in patients with liver cirrhosis and hepatocellular carcinoma-a pilot study. Aliment Pharmacol Ther 2012;35:83-91.

81 Lata J: Chronic liver diseases as liver tumor precursors. Dig Dis 2010;28:596-599. 